\title{
MULTIVARIATE SUBEXPONENTIAL DISTRIBUTIONS AND RANDOM SUMS OF RANDOM VECTORS
}

\author{
E. OMEY,$^{*}$ European University College Brussels \\ F. MALLOR ${ }^{* *}$ AND \\ J. SANTOS, ${ }^{* * *}$ Public University of Navarre
}

\begin{abstract}
Let $F(\boldsymbol{x})$ denote a distribution function in $\mathbb{R}^{d}$ and let $F^{* n}(\boldsymbol{x})$ denote the $n$th convolution power of $F(\boldsymbol{x})$. In this paper we discuss the asymptotic behaviour of $1-F^{* n}(\boldsymbol{x})$ as $\boldsymbol{x}$ tends to $\infty$ in a certain prescribed way. It turns out that in many cases $1-F^{* n}(\boldsymbol{x}) \sim$ $n(1-F(\boldsymbol{x}))$. To obtain results of this type, we introduce and use a form of subexponential behaviour, thereby extending the notion of multivariate regular variation. We also discuss subordination, in which situation the index $n$ is replaced by a random index $N$.
\end{abstract}

Keywords: Subexponential distribution; regular variation; random sum

2000 Mathematics Subject Classification: Primary 26A12

Secondary 60E99; 60K99

\section{Introduction}

Let $F(\boldsymbol{x})$ denote a distribution function (DF) in $\mathbb{R}^{d}$ with $F(\mathbf{0}+)=0$ and $F(\boldsymbol{x})<1$ for all $\boldsymbol{x} \in \mathbb{R}^{d}$. Let $\boldsymbol{X}, \boldsymbol{X}_{1}, \boldsymbol{X}_{2}, \ldots, \boldsymbol{X}_{n}$ denote independent random vectors with common DF $F(\boldsymbol{x})$. Let $\boldsymbol{S}(\mathbf{0})=0$ and, for $n \geq 1$, let $\boldsymbol{S}(n)=\boldsymbol{S}(n-1)+\boldsymbol{X}_{n}$. The marginal partial sums will be denoted by $S^{i}(n)$, i.e. $S(n)=\left(S^{1}(n), S^{2}(n), \ldots, S^{d}(n)\right)$.

Let $N$ denote an integer-valued random variable independent of $\boldsymbol{X}$ with probability distribution $\mathrm{P}(N=n)=p_{n}$. The new random variable $\boldsymbol{S}(N)$ has $\mathrm{DF}$

$$
W(\boldsymbol{x})=\mathrm{P}(\boldsymbol{S}(N) \leq \boldsymbol{x})=\sum_{n=0}^{\infty} p_{n} \mathrm{P}(\boldsymbol{S}(n) \leq \boldsymbol{x})=\sum_{n=0}^{\infty} p_{n} F^{* n}(\boldsymbol{x}),
$$

where $F^{* n}(\boldsymbol{x})$ denotes the $n$-fold convolution of $F(\boldsymbol{x}), F^{* 0}(\boldsymbol{x})$ denoting the unit mass at 0 . The DF $W(\boldsymbol{x})$ is said to be subordinate to $F(\boldsymbol{x})$ with subordinator $\left\{p_{n}\right\}$. In the special case where $\mathrm{P}(N=n)=1$, we have $W(\boldsymbol{x})=\mathrm{P}(\boldsymbol{S}(n) \leq \boldsymbol{x})=F^{* n}(\boldsymbol{x})$.

In a more general approach, let $N=\left(N_{1}, N_{2}, \ldots, N_{d}\right)$ denote a vector of integer-valued random variables independent of $\boldsymbol{X}$. A new random variable $\boldsymbol{S}(\boldsymbol{N})$ is defined as follows:

$$
\boldsymbol{S}(\boldsymbol{N})=\left(S^{1}\left(N_{1}\right), S^{2}\left(N_{2}\right), \ldots, S^{d}\left(N_{d}\right)\right) .
$$

The DF of $S(N)$ will be denoted by $K(\boldsymbol{x})=\mathrm{P}(\boldsymbol{S}(\boldsymbol{N}) \leq \boldsymbol{x})$.

\footnotetext{
Received 5 October 2005; revision received 24 May 2006.

* Postal address: Department of Mathematics and Statistics, European University College Brussels, Stormstraat 2, 1000 Brussels, Belgium.

** Postal address: Department of Statistics and Operations Research, Public University of Navarre, Campus Arrosadia, 31006 Pamplona, Spain. Email address: mallor@unavarra.es

*** Current address: Los Alderetes 14, 4 3, 14004 Cordoba, Spain.
} 
In this paper we discuss the relation between the asymptotic behaviour of the tails of the distributions introduced above. From now on we use the shorthand notation $\bar{F}=1-F$. It turns out that, as in the univariate case, there are many cases in which $\overline{F^{* n}}(\boldsymbol{x})$ asymptotically behaves like $n \bar{F}(\boldsymbol{x})$ and $\bar{W}(\boldsymbol{x})$ behaves like $\mathrm{E}(N) \bar{F}(\boldsymbol{x})$. It also turns out that the asymptotic behaviour of $\bar{K}(\boldsymbol{x})$ is determined by that of $\bar{F}(\boldsymbol{x})$ and its marginals. To specify these relations, we present a form of multivariate subexponentiality.

The paper is organized as follows. In Section 2 we briefly recall some basic properties and definitions concerning univariate subexponential DFs and introduce multivariate subexponential DFs. In Section 3 we state our main results concerning the asymptotic behaviour of $\overline{F^{* n}}(\boldsymbol{x})$, $\bar{W}(\boldsymbol{x})$, and $\bar{K}(\boldsymbol{x})$. In Section 4 we briefly discuss the relation with regular variation, and finish the paper with some applications and final remarks.

\section{Subexponential distributions}

\subsection{Univariate subexponential distributions}

In the one-dimensional case, let $F(x)$ denote the DF of a positive random variable $X$ such that

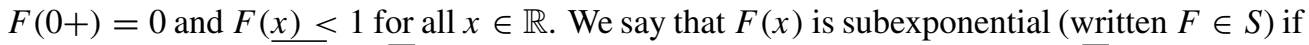
it satisfies $\lim _{x \rightarrow \infty} \overline{F^{* 2}}(x) / \bar{F}(x)=2$. It is well known that $F \in S$ implies that $\bar{F} \in L$, where $L$ denotes the class of positive, measurable functions $u(x)$ such that $\lim _{x \rightarrow \infty} u(x-y) / u(x)=1$ for all $y \in \mathbb{R}$. The converse statement $(\bar{F} \in L$ implies $F \in S)$ is false. Further basic properties can be found in, for example, [7], [8], [37], and [12]-[16]. A survey of results was given by Goldie and Klüppelberg in [18].

Extending the class $S$, Chistyakov [4] and Chover et al. [5], [6] introduced the class $S_{\gamma}$, $\gamma \geq 0$. We say that $F$ belongs to the class $S_{\gamma}$ if it satisfies the following properties.

- $f(s)=\mathrm{E}(\exp (-s X))<\infty$ for $s \geq-\gamma$.

- $\bar{F} \in L_{\gamma}$, where $L_{\gamma}$ denotes the class of positive, measurable functions $u(x)$ such that, as $x \rightarrow \infty, u(x-y) / u(x) \rightarrow \exp (\gamma y)$ for all $y \in \mathbb{R}$.

- $F$ satisfies $\overline{F^{* 2}}(x) / \bar{F}(x) \rightarrow 2 f(-\gamma)$ as $x \rightarrow \infty$.

The class $S$ is large and contains, for example, all DFs with regularly varying tails. For a review of regular variation, we refer the reader to the books of Bingham et al. [3], Geluk and de Haan [17], and Seneta [35].

Note that $S_{0}=S$ and that $L_{0}=L$. The next result illustrates the use of subexponential distributions in the context of subordinated DFs in the univariate case.

Lemma 1. ([8], [16].) Let $P(z)=\mathrm{E}\left(z^{N}\right)$ and $\gamma \geq 0$.

(i) Suppose that $P(z)$ is analytic at $z=1$. Then $F \in S$ if and only if $\bar{W}(x) / \bar{F}(x) \rightarrow \mathrm{E}(N)$ as $x \rightarrow \infty$, and both statements imply that $W \in S$.

(ii) Suppose that $P(z)$ is analytic at $z=f(-\gamma)$. If $F \in S_{\gamma}$ then $\bar{W}(x) / \bar{F}(x) \rightarrow P^{\prime}(f(-\gamma))$ as $x \rightarrow \infty$ and $W \in S_{\gamma}$.

Lemma 1(i) shows that, for $F \in S$, we have $\mathrm{P}(S(n)>x) \sim n \mathrm{P}(X>x)$, i.e. the tail distribution of the partial sums $S(n)=\sum_{i=1}^{n} X_{i}$ asymptotically behaves like $n$ times the tail distribution of $X$. To prove a multivariate analogue of this result, we shall frequently use the following result concerning the classes $S$ and $S_{\gamma}$. The result is well known if $\gamma=0$ and easily extends to cases where $\gamma>0$. 
Lemma 2. ([8], [14].) Let $F, G$, and $H$ denote the DFs of positive random variables and assume that $F \in S_{\gamma}$. Let $h(s)$ and $g(s)$ denote the Laplace-Stieltjes transforms of $H$ and $G$, respectively. If $\bar{G}(x) / \bar{F}(x) \rightarrow \alpha$ and $\bar{H}(x) / \bar{F}(x) \rightarrow \beta$ as $x \rightarrow \infty$, then

1. $\overline{G * H}(x) / \bar{F}(x) \rightarrow \alpha h(-\gamma)+\beta g(-\gamma)$ as $x \rightarrow \infty$,

2. $\int_{0}^{x / 2} \bar{G}(x-u) \mathrm{d} H(u) / \bar{F}(x) \rightarrow \alpha h(-\gamma)$ as $x \rightarrow \infty$,

3. $\bar{G}(x / 2) \bar{H}(x / 2) / \bar{F}(x) \rightarrow 0$ as $x \rightarrow \infty$.

\subsection{Multivariate subexponential distributions}

To generalize the one-dimensional results we introduce several types of multivariate subexponential behaviour. For $\boldsymbol{x}=\left(x_{1}, x_{2}, \ldots, x_{d}\right) \in \mathbb{R}^{d}$, we set $x_{\min }=\min \left(x_{1}, x_{2}, \ldots, x_{d}\right)$. In [28] the following classes of DF were defined.

Definition 1. $F \in S\left(\mathbb{R}^{d}\right)$ if and only if

$$
\lim _{t \rightarrow \infty} \frac{\overline{F^{* 2}}(t \boldsymbol{x})}{\bar{F}(t \boldsymbol{x})}=2 \quad \text { for all } \boldsymbol{x}>\mathbf{0} \text { with } x_{\min }<\infty .
$$

Definition 2. $\bar{F} \in L\left(\mathbb{R}^{d}\right)$ if and only if

$$
\lim _{t \rightarrow \infty} \frac{\bar{F}(t \boldsymbol{x}-\boldsymbol{a})}{\bar{F}(t \boldsymbol{x})}=1 \quad \text { for all } \boldsymbol{x}>\mathbf{0} \text { with } x_{\min }<\infty \text { and all } \boldsymbol{a} \geq \mathbf{0} .
$$

Note that in (1) and (2) we assume that the marginals are subexponential and that the marginals are in class $L$, respectively. In [28] it was proved that $F \in S\left(\mathbb{R}^{d}\right)$ implies that $F \in L\left(\mathbb{R}^{d}\right)$. Moreover, these statements are almost equivalent, and the multivariate analogue of Lemma 1 holds.

Theorem 1. ([28].) (i) We have $F \in S\left(\mathbb{R}^{d}\right)$ if and only if $\bar{F} \in L\left(\mathbb{R}^{d}\right)$ and the marginals, $F_{i}$, of $F$ are subexponential in $\mathbb{R}$.

(ii) If $\bar{F} \in S\left(\mathbb{R}^{d}\right)$ and if $P(z)=\mathrm{E}\left(z^{N}\right)$ is analytic at $z=1$, then

$$
\lim _{t \rightarrow \infty} \frac{\bar{W}(t \boldsymbol{x}-\boldsymbol{a})}{\bar{F}(t \boldsymbol{x})}=\mathrm{E}(N) \quad \text { for all } \boldsymbol{x}>\mathbf{0} \text { with } x_{\min }<\infty \text { and all } \boldsymbol{a} \geq \mathbf{0} .
$$

By replacing the limits in (1) and (2), we extend the classes $S\left(\mathbb{R}^{d}\right)$ and $L\left(\mathbb{R}^{d}\right)$ in an obvious way.

Definition 3. $F \in S\left(\mathbb{R}^{d}, \alpha\right)$ if and only if

$$
\lim _{t \rightarrow \infty} \frac{\overline{F^{* 2}}(t \boldsymbol{x})}{\bar{F}(t \boldsymbol{x})}=\alpha(\boldsymbol{x}) \quad \text { for all } \boldsymbol{x}>\mathbf{0} \text { with } x_{\min }<\infty .
$$

Definition 4. $\bar{F} \in L\left(\mathbb{R}^{d}, v\right)$ if and only if

$$
\lim _{t \rightarrow \infty} \frac{\bar{F}(t \boldsymbol{x}-\boldsymbol{a})}{\bar{F}(t \boldsymbol{x})}=v(\boldsymbol{x}, \boldsymbol{a}) \quad \text { for all } \boldsymbol{x}>\mathbf{0} \text { with } x_{\min }<\infty \text { and all } \boldsymbol{a} \geq \mathbf{0} .
$$


Definition 5. $\bar{F} \in L^{*}\left(\mathbb{R}^{d}, v\right)$ if and only if

$$
\lim _{t \rightarrow \infty} \frac{\bar{F}(t \boldsymbol{x}-\boldsymbol{b}-\boldsymbol{a})}{\bar{F}(t \boldsymbol{x}-\boldsymbol{b})}=v(\boldsymbol{x}, \boldsymbol{a}) \quad \text { for all } \boldsymbol{x}>\mathbf{0} \text { with } x_{\min }<\infty \text { and all } \boldsymbol{a}, \boldsymbol{b} \geq \mathbf{0} .
$$

Note that in Definitions 3-5 we assume that the marginals satisfy the same type of relation. For example, for the $i$ th marginal $(i=1,2, \ldots, d)$, Definition 3 reads as follows: for all $x_{i}>0$ we have

$$
\lim _{t \rightarrow \infty} \frac{\overline{F^{* 2}}\left(t x_{i}\right)}{\bar{F}\left(t x_{i}\right)}=\alpha_{i}\left(x_{i}\right)
$$

In a similar way, we will denote the limits for the marginals in Definitions 4 and 5 by $v_{i}\left(x_{i}, a_{i}\right)$. We use similar conventions in the definitions below.

\subsection{Other normalizations}

In our definitions, we consider limits along lines of the form $t \boldsymbol{x}$. Alternatively, we can discuss limits along curves of the form $\boldsymbol{c}(t) * \boldsymbol{x}=\left(c_{1}(t) x_{1}, c_{2}(t) x_{2}, \ldots, c_{d}(t) x_{d}\right)$, where for each $i$ we have $c_{i}(t) \rightarrow \infty$ as $t \rightarrow \infty$.

Definition 6. $F \in S^{c}\left(\mathbb{R}^{d}, v\right)$ if and only if

$$
\lim _{t \rightarrow \infty} \frac{\overline{F^{* 2}}(\boldsymbol{c}(t) * \boldsymbol{x})}{\bar{F}(\boldsymbol{c}(t) * \boldsymbol{x})}=v(\boldsymbol{x}) \quad \text { for all } \boldsymbol{x}>\mathbf{0} \text { with } x_{\min }<\infty .
$$

The class $L^{c}\left(\mathbb{R}^{d}, v\right)$ can be defined in a similar way. In most parts of our paper, this definition brings nothing new; however, when we need more precise information related to the marginal distributions, Definition 6 may be useful (see Section 3.3, below).

Consider, for example, a DF of the form $\bar{F}(x, y) \approx \overline{F_{1}}(x)+\overline{F_{2}}(y)$, where $\overline{F_{1}}(x) \approx 1 / x$ and $\overline{F_{2}}(y) \approx 1 / y^{2}$. In this case, we have $t \bar{F}(t x-a, \sqrt{t} y-b) \rightarrow 1 / x+1 / y^{2}$ and $\bar{F} \in L^{c}\left(\mathbb{R}^{2}, v\right)$ with $\left(c_{1}(t), c_{2}(t)\right)=(t, \sqrt{t})$ and $v=1$. We also have $\overline{F_{1}}(t x) / \bar{F}(t x, \sqrt{t} y) \rightarrow y^{2} /\left(x+y^{2}\right)$.

For this example we also have $t \bar{F}(t x-a, t y-b) \rightarrow 1 / x$ and $\bar{F} \in L\left(\mathbb{R}^{2}\right)$, in which case $\overline{F_{1}}(t x) / \bar{F}(t x, t y) \rightarrow 1$.

Remark 1. In [9] Cline and Resnick introduced and studied a form of multivariate subexponential distribution. Their approach is formulated in terms of vague convergence and uses point process arguments. Slightly adapted to our notation, in their definition of a class $L$ Cline and Resnick assumed that $\lim _{t \rightarrow \infty} t \bar{F}(\boldsymbol{r}(t)+\boldsymbol{a})=v(\boldsymbol{a})$ for all $\boldsymbol{a}$, where $\boldsymbol{r}(t)=$ $\left(r_{1}(t), r_{2}(t), \ldots, r_{d}(t)\right)$ and $r_{i}(t) \rightarrow \infty, i=1,2, \ldots, d$, as $t \rightarrow \infty$. The definition automatically implies that, for each marginal, we have

$$
\lim _{t \rightarrow \infty} t \overline{F_{i}}\left(r_{i}(t)+a\right)=v_{i}(a) \text { for almost all } a,
$$

and this relation determines the normalizing functions $r_{i}(t)$. Moreover, if $v_{i}(a) \neq 0$ then

$$
\lim _{t \rightarrow \infty} \frac{\bar{F}(\boldsymbol{r}(t)+\boldsymbol{a})}{\overline{F_{i}}\left(r_{i}(t)+a\right)}=v^{*}(\boldsymbol{a}) \text { for all } \boldsymbol{a},
$$

and this relation determines a relationship between the joint DF and one (or more) of the marginals. In our definition we do not make such an assumption at first. Only in the discussion of $\boldsymbol{S}(\boldsymbol{N})$ (see Section 3.3) do we have to make a similar assumption. 


\section{Main results}

In this section we start to obtain some useful information about the limit function in Definitions 4 and 5. In Section 3.2 we determine the limit function in Definition 3. In Section 3.3 we discuss the multivariate analogues of Lemmas 1 and 2. In Section 3.4 we discuss random vectors of the form $\left(S^{1}\left(N_{1}\right), S^{2}\left(N_{2}\right)\right)$.

\subsection{The limit function $v(x, a)$}

In general it is hard to calculate $v(\boldsymbol{x}, \boldsymbol{a})$ explicitly. In the following lemma we obtain some characteristics of this limit function.

Lemma 3. (i) If $\bar{F} \in L\left(\mathbb{R}^{d}, v\right)$ then $\mathrm{E}(v(\boldsymbol{x}, \boldsymbol{X}))<\infty$.

(ii) If $\bar{F} \in L^{*}\left(\mathbb{R}^{d}, v\right)$ then $v(\boldsymbol{x}, \boldsymbol{a})=\exp \left(\sum_{i=1}^{d} A_{i}(\boldsymbol{x}) a_{i}\right)$ for some functions $A_{i}(\boldsymbol{x})$.

(iii) Suppose that $\bar{F} \in L^{*}\left(\mathbb{R}^{d}, v\right)$ and that, for all $\boldsymbol{a} \geq \mathbf{0}, \boldsymbol{b} \geq \mathbf{0}$, and $\boldsymbol{x}>\mathbf{0}$ with $x_{\min }<\infty$, $\bar{H}$ satisfies

$$
\lim _{t \rightarrow \infty} \frac{\bar{H}(t \boldsymbol{x}-\boldsymbol{a}-\boldsymbol{b})}{\bar{F}(t \boldsymbol{x}-\boldsymbol{a})}=\beta(\boldsymbol{x}, \boldsymbol{b})
$$

for some real function $\beta(\boldsymbol{x}, \boldsymbol{b})$. Then $\beta(\boldsymbol{x}, \boldsymbol{a})=B(\boldsymbol{x}) v(\boldsymbol{x}, \boldsymbol{a})$ for some function $B(\boldsymbol{x})$.

Proof. (i) If $\bar{F} \in L\left(\mathbb{R}^{d}, v\right)$ then, for each marginal $F_{i}$, we have

$$
\lim _{t \rightarrow \infty} \frac{\overline{F_{i}}(t x-a)}{\overline{F_{i}}(t x)}=v_{i}(x, a)
$$

for some limit function $v_{i}$. Replacing $t x$ by $t$, we find that

$$
\lim _{t \rightarrow \infty} \frac{\overline{F_{i}}(t-a)}{\overline{F_{i}}(t)}=v_{i}(x, a),
$$

and we see that $v_{i}(x, a) \equiv v_{i}(a)$ is independent of $x$. From

$$
\overline{F_{i}}(x-a-b)=\frac{\overline{F_{i}}(x-a-b)}{\overline{F_{i}}(x-a)} \overline{F_{i}}(x-a),
$$

it follows that $v_{i}(a+b)=v_{i}(a) v_{i}(b)$ and, consequently, that $v_{i}(a)=\exp (\lambda(i) a)$ for some real number $\lambda(i) \geq 0$. Therefore, we find that $\overline{F_{i}} \in L_{\lambda(i)}$ for each marginal. For $v(\boldsymbol{x}, \boldsymbol{a})$ we have the following information. Using $\bar{F}(\boldsymbol{x}) \leq \sum_{i=1}^{d} \overline{F_{i}}\left(x_{i}\right)$ and $\overline{F_{i}}\left(x_{i}\right) \leq \bar{F}(\boldsymbol{x})$, we have

$$
\frac{\bar{F}(t \boldsymbol{x}-\boldsymbol{a})}{\bar{F}(t \boldsymbol{x})} \leq \sum_{i=1}^{d} \frac{\bar{F}_{i}\left(t x_{i}-a_{i}\right)}{\bar{F}_{i}\left(t x_{i}\right)}
$$

and it follows that

$$
v(\boldsymbol{x}, \boldsymbol{a}) \leq \sum_{i=1}^{d} v_{i}\left(a_{i}\right)=\sum_{i=1}^{d} \exp \left(\lambda(i) a_{i}\right) .
$$

If $F \in S\left(\mathbb{R}^{d}, \alpha\right)$ and $\bar{F} \in L\left(\mathbb{R}^{d}, v\right)$ then we find that $F_{i} \in S_{\lambda(i)}$ for each of the marginals, and the previous analysis shows that

$$
\mathrm{E} v(\boldsymbol{x}, \boldsymbol{X}) \leq \sum_{i=1}^{d} f_{i}(-\lambda(i))<\infty, \quad \text { where } f_{i}(s)=\mathrm{E}\left(\exp \left(-s X_{i}\right)\right)
$$


(ii) If $\bar{F} \in L^{*}\left(\mathbb{R}^{d}, v\right)$, we use

$$
\bar{F}(t \boldsymbol{x}-\boldsymbol{a}-\boldsymbol{b})=\frac{\bar{F}(t \boldsymbol{x}-\boldsymbol{a}-\boldsymbol{b})}{\bar{F}(t \boldsymbol{x}-\boldsymbol{a})} \bar{F}(t \boldsymbol{x}-\boldsymbol{a})
$$

to find that $v(\boldsymbol{x}, \boldsymbol{a}+\boldsymbol{b})=v(\boldsymbol{x}, \boldsymbol{a}) v(\boldsymbol{x}, \boldsymbol{b})$. By taking $\boldsymbol{a}=(a, 0,0, \ldots, 0)$ and $\boldsymbol{b}=(b, 0$, $0, \ldots, 0)$, it follows that

$$
v(\boldsymbol{x},(a+b, 0,0, \ldots, 0))=v(\boldsymbol{x},(a, 0,0, \ldots, 0)) v(\boldsymbol{x},(b, 0,0, \ldots, 0))
$$

and, consequently, that $v(\boldsymbol{x},(a, 0,0, \ldots, 0))=\exp \left(A_{1}(\boldsymbol{x}) a\right)$ for some function $A_{1}(\boldsymbol{x})$. In a similar way we find, for the $i$ th component, that

$$
v(\boldsymbol{x},(0, \ldots, a, \ldots, 0))=\exp \left(A_{i}(\boldsymbol{x}) a\right), \quad 1 \leq i \leq d .
$$

Finally, by using $\boldsymbol{a}=\left(a_{1}, 0,0, \ldots, 0\right)+\left(0, a_{2}, 0, \ldots, 0\right)+\cdots+\left(0,0, \ldots, a_{d}\right)$, we find that

$$
v(\boldsymbol{x}, \boldsymbol{a})=\exp \left(\sum_{i=1}^{d} A_{i}(\boldsymbol{x}) a_{i}\right)
$$

(iii) We write

$$
\frac{\bar{H}(t \boldsymbol{x}-\boldsymbol{a}-\boldsymbol{b})}{\bar{F}(t \boldsymbol{x})}=\frac{\bar{H}(t \boldsymbol{x}-\boldsymbol{a}-\boldsymbol{b})}{\bar{F}(t \boldsymbol{x}-\boldsymbol{a})} \frac{\bar{F}(t \boldsymbol{x}-\boldsymbol{a})}{\bar{F}(t \boldsymbol{x})}
$$

to see that $\beta(\boldsymbol{x}, \boldsymbol{a}+\boldsymbol{b})=\beta(\boldsymbol{x}, \boldsymbol{b}) v(\boldsymbol{x}, \boldsymbol{a})$. To complete the proof, now take $\boldsymbol{b}=\mathbf{0}$.

\subsection{Closure property}

To prove a multidimensional analogue of Lemma 2, we need the following lemma, which extends [28, Proposition 3.8].

Lemma 4. (i) Let $\boldsymbol{U}, \boldsymbol{V} \in \mathbb{R}^{d}$ denote independent, nonnegative random vectors with DFs $G(\cdot)$ and $H(\cdot)$, respectively. Then, for all $\boldsymbol{x} \geq \mathbf{0}$, we have

$$
1-G * H(x)-J(1)-J(2) \leq \bar{G}(x / 2) \bar{H}(x / 2),
$$

where

$$
J(1)=\int_{0}^{\boldsymbol{x} / 2} \bar{H}(\boldsymbol{x}-\boldsymbol{u}) \mathrm{d} G(\boldsymbol{u}) \text { and } J(2)=\int_{0}^{\boldsymbol{x} / 2} \bar{G}(\boldsymbol{x}-\boldsymbol{u}) \mathrm{d} H(\boldsymbol{u}) .
$$

(ii) Let $m<d$ and suppose that $\boldsymbol{U} \in \mathbb{R}^{m}$ and $\boldsymbol{V} \in \mathbb{R}^{d}$ are nonnegative, independent random vectors with DFs $G(\cdot)$ and $H(\cdot)$, respectively. Let $\boldsymbol{U}^{\circ}=(\boldsymbol{U}, 0) \in \mathbb{R}^{d}$ and let $G^{\circ}(\cdot)$ denote its DF. Then, for all $\boldsymbol{x} \in \mathbb{R}^{d}$ with $\boldsymbol{x} \geq \mathbf{0}$, we have

$$
1-G^{\circ} * H(\boldsymbol{x})-J(1)-J(2) \leq \bar{G}\left(\boldsymbol{x}_{(m)} / 2\right) \bar{H}(\boldsymbol{x} / 2),
$$

where $\boldsymbol{x}_{(m)}=\left(x_{1}, x_{2}, \ldots, x_{m}\right)$,

$$
J(1)=\int_{0}^{x / 2} \bar{H}(x-u) \mathrm{d} G^{\circ}(\boldsymbol{u}), \quad \text { and } \quad J(2)=\int_{0}^{x / 2} \overline{G^{\circ}}(\boldsymbol{x}-\boldsymbol{u}) \mathrm{d} H(\boldsymbol{u}) .
$$


Proof. (i) Observe that

$$
\begin{aligned}
& J(1)=\mathrm{P}(\boldsymbol{U} \leq \boldsymbol{x} / 2)-\mathrm{P}(\boldsymbol{U}+\boldsymbol{V} \leq \boldsymbol{x}, \boldsymbol{U} \leq \boldsymbol{x} / 2), \\
& J(2)=\mathrm{P}(\boldsymbol{V} \leq \boldsymbol{x} / 2)-\mathrm{P}(\boldsymbol{U}+\boldsymbol{V} \leq \boldsymbol{x}, \boldsymbol{V} \leq \boldsymbol{x} / 2),
\end{aligned}
$$

and $G * H(\boldsymbol{x})=\mathrm{P}(\boldsymbol{U}+\boldsymbol{V} \leq \boldsymbol{x})=P_{1}+P_{2}+P_{3}+P_{4}$, where

$$
\begin{aligned}
& P_{1}=\mathrm{P}(\boldsymbol{U}+\boldsymbol{V} \leq \boldsymbol{x}, \boldsymbol{U} \leq \boldsymbol{x} / 2, \boldsymbol{V} \leq \boldsymbol{x} / 2), \\
& P_{2}=\mathrm{P}\left(\boldsymbol{U}+\boldsymbol{V} \leq \boldsymbol{x},\{\boldsymbol{U} \leq \boldsymbol{x} / 2\}^{\mathrm{c}}, \boldsymbol{V} \leq \boldsymbol{x} / 2\right), \\
& P_{3}=\mathrm{P}\left(\boldsymbol{U}+\boldsymbol{V} \leq \boldsymbol{x},\{\boldsymbol{V} \leq \boldsymbol{x} / 2\}^{\mathrm{c}}, \boldsymbol{U} \leq \boldsymbol{x} / 2\right), \\
& P_{4}=\mathrm{P}\left(\boldsymbol{U}+\boldsymbol{V} \leq \boldsymbol{x},\{\boldsymbol{V} \leq \boldsymbol{x} / 2\}^{\mathrm{c}},\{\boldsymbol{U} \leq \boldsymbol{x} / 2\}^{\mathrm{c}}\right) .
\end{aligned}
$$

Using $\mathrm{P}(\boldsymbol{U}+\boldsymbol{V} \leq \boldsymbol{x}, \boldsymbol{V} \leq \boldsymbol{x} / 2)-P_{2}=P_{1}$ and $\mathrm{P}(\boldsymbol{U}+\boldsymbol{V} \leq \boldsymbol{x}, \boldsymbol{U} \leq \boldsymbol{x} / 2)-P_{3}=P_{1}$, we find that

$$
I=1-G * H(\boldsymbol{x})-J(1)-J(2)=1-\mathrm{P}(\boldsymbol{V} \leq \boldsymbol{x} / 2)-\mathrm{P}(\boldsymbol{U} \leq \boldsymbol{x} / 2)+P_{1}-P_{4} .
$$

Since $P_{1}=\mathrm{P}(\boldsymbol{U} \leq \boldsymbol{x} / 2, \boldsymbol{V} \leq \boldsymbol{x} / 2)=\mathrm{P}(\boldsymbol{U} \leq \boldsymbol{x} / 2) \mathrm{P}(\boldsymbol{V} \leq \boldsymbol{x} / 2)$, it follows that

$$
I=\bar{G}(x / 2) \bar{H}(x / 2)-P_{4},
$$

using $0 \leq P_{4} \leq \mathrm{P}\left(\{\boldsymbol{V} \leq \boldsymbol{x} / 2\}^{\mathrm{c}},\{\boldsymbol{U} \leq \boldsymbol{x} / 2\}^{\mathrm{c}}\right)=\bar{G}(\boldsymbol{x} / 2) \bar{H}(\boldsymbol{x} / 2)$.

(ii) The proof follows from part (i).

Remark 2. In part (ii) we can rewrite $J(1)$ and $J(2)$ as, respectively,

$$
J(1)=\int_{0}^{x_{1} / 2} \int_{0}^{x_{2} / 2} \cdots \int_{0}^{x_{m} / 2} \bar{H}\left(\boldsymbol{x}_{(m)}-\boldsymbol{u}_{(m)}, x_{m+1}, \ldots, x_{d}\right) \mathrm{d} G(\boldsymbol{u})
$$

and

$$
J(2)=\int_{0}^{x_{1} / 2} \int_{0}^{x_{2} / 2} \cdots \int_{0}^{x_{d} / 2} \bar{G}\left(\boldsymbol{x}_{(m)}-\boldsymbol{u}_{(m)}\right) \mathrm{d} H(\boldsymbol{u}) .
$$

Now we come to the main result of this section. Let $\boldsymbol{X} \in \mathbb{R}^{d}$ denote a nonnegative random vector with DF $F(\boldsymbol{x})$, and let $\boldsymbol{U}$ and $\boldsymbol{V}$ denote nonnegative random vectors with respective DFs $G$ and $H$ as in Lemma 3. We impose the following conditions.

(C1) For some real function $v$, we have $\bar{F}(\boldsymbol{x}) \in L\left(\mathbb{R}^{d}, v\right)$.

(C2) The marginals, $F_{i}$, of $F$ satisfy $F_{i}(x) \in S_{\lambda(i)}$.

(C3) For all $\boldsymbol{a} \geq \boldsymbol{0}$, all $\boldsymbol{x}>\boldsymbol{0}$ with $x_{\min }<\infty$, and some real function $\alpha$, we have

$$
\lim _{t \rightarrow \infty} \frac{\overline{G^{\circ}}(t \boldsymbol{x}-\boldsymbol{a})}{\bar{F}(t \boldsymbol{x})}=\lim _{t \rightarrow \infty} \frac{\overline{\boldsymbol{G}}\left(t \boldsymbol{x}_{(m)}-\boldsymbol{a}_{(m)}\right)}{\bar{F}(t \boldsymbol{x})}=\alpha\left(\boldsymbol{x}, \boldsymbol{a}_{(m)}\right) .
$$

(C4) For all $\boldsymbol{a} \geq \mathbf{0}$, all $\boldsymbol{x}>\boldsymbol{0}$ with $x_{\min }<\infty$, and some real function $\beta$, we have

$$
\lim _{t \rightarrow \infty} \frac{\bar{H}(t \boldsymbol{x}-\boldsymbol{a})}{\bar{F}(t \boldsymbol{x})}=\beta(\boldsymbol{x}, \boldsymbol{a}) .
$$


Note that (C3) and (C4) imply that, for the marginals of $H$ and $G$, we have

$$
\lim _{t \rightarrow \infty} \frac{\overline{G_{i}}(t x-a)}{\overline{F_{i}}(t x)}=C_{i} \exp (\lambda(i) a)=\alpha_{i}(a), \quad 1 \leq i \leq m,
$$

and

$$
\lim _{t \rightarrow \infty} \frac{\overline{H_{i}}(t x-a)}{\overline{F_{i}}(t x)}=C_{i}^{\prime} \exp (\lambda(i) a)=\beta_{i}(a), \quad 1 \leq i \leq d,
$$

where $C_{i}$ and $C_{i}^{\prime}$ are nonnegative constants.

In the next theorem we provide conditions under which (C3) and (C4) imply that $\overline{G * H}$ satisfies a relation of the same form. First we consider the case where $\boldsymbol{U}, \boldsymbol{V} \in \mathbb{R}^{d}$ (meaning that $G=G^{\circ}$ ).

Theorem 2. (i) Suppose that $m=d$ and that (C2)-(C4) hold. Then, for all $\boldsymbol{a} \geq \mathbf{0}$ and all $\boldsymbol{x}>\mathbf{0}$ with $x_{\min }<\infty$, we have

$$
\lim _{t \rightarrow \infty} \frac{\overline{G * H}(t \boldsymbol{x}-\boldsymbol{a})}{\bar{F}(t \boldsymbol{x})}=\mathrm{E}(\alpha(\boldsymbol{x}, \boldsymbol{a}+\boldsymbol{V}))+\mathrm{E}(\beta(\boldsymbol{x}, \boldsymbol{a}+\boldsymbol{U})) .
$$

(ii) For vectors of different dimensions, we find that if (C2)-(C4) hold then, for all $\boldsymbol{a} \geq \mathbf{0}$ and all $\boldsymbol{x}>\boldsymbol{0}$ with $x_{\min }<\infty$, we have

$$
\lim _{t \rightarrow \infty} \frac{\overline{G^{\circ} * H}(t \boldsymbol{x}-\boldsymbol{a})}{\bar{F}(t \boldsymbol{x})}=\mathrm{E}\left(\alpha\left(\boldsymbol{x}, \boldsymbol{a}_{(m)}+\boldsymbol{V}_{(m)}\right)\right)+\mathrm{E}(\beta(\boldsymbol{x}, \boldsymbol{a}+(\boldsymbol{U}, 0))) .
$$

Proof. (i) The proof is based on Lemma 3. Let $\boldsymbol{z}=\boldsymbol{t} \boldsymbol{x}-\boldsymbol{a}$ with $t$ large. We start with the term $\bar{G}(z / 2) \bar{H}(z / 2)$. We have $\bar{G}(z / 2) \leq \sum_{i=1}^{d} \overline{G_{i}}\left(z_{i} / 2\right)$. Using $(\mathrm{C} 3)$, we obtain $\bar{G}(z / 2)=$ $O(1) \sum_{i=1}^{d} \overline{F_{i}}\left(t x_{i} / 2\right)$. In a similar way, using (C4), we have $\bar{H}(z / 2)=O(1) \sum_{i=1}^{d} \overline{F_{i}}\left(t x_{i} / 2\right)$. From (C2) and Lemma 2, we also have $\overline{F_{i}}\left(t x_{i} / 2\right)=o(1) \sqrt{\overline{F_{i}}\left(t x_{i}\right)}$. Using $\overline{F_{i}}\left(t x_{i}\right) \leq \bar{F}(t \boldsymbol{x})$ we readily find that $\bar{G}(z / 2) \bar{H}(z / 2)=o(1) \bar{F}(t \boldsymbol{x})$. Then, applying Lemma 3, we obtain

$$
\lim _{t \rightarrow \infty}\left(\frac{\overline{G * H}(t \boldsymbol{x}-\boldsymbol{a})}{\bar{F}(t \boldsymbol{x})}-\frac{J(1)}{\bar{F}(t \boldsymbol{x})}-\frac{J(2)}{\bar{F}(t \boldsymbol{x})}\right)=0 .
$$

Now we deal with the term

$$
T(1)=\frac{J(1)}{\bar{F}(t \boldsymbol{x})}=\int_{\mathbf{0}}^{z / 2} \frac{\bar{H}(z-\boldsymbol{U})}{\bar{F}(t \boldsymbol{x})} \mathrm{d} G(\boldsymbol{U}) .
$$

We note the following facts.

1. Using (C4), we have $\lim _{t \rightarrow \infty} \bar{H}(z-\boldsymbol{u}) / \bar{F}(t \boldsymbol{x})=\beta(\boldsymbol{x}, \boldsymbol{a}+\boldsymbol{u})$.

2. Using $\bar{H}(z-\boldsymbol{u}) \leq \sum_{i=1}^{d} \overline{H_{i}}\left(z_{i}-u_{i}\right)$ and $\overline{F_{i}}\left(t x_{i}\right) \leq \bar{F}(t \boldsymbol{x})$, we have

$$
\frac{\bar{H}(\boldsymbol{z}-\boldsymbol{u})}{\bar{F}(t \boldsymbol{x})} 1_{\{\mathbf{0} \leq \boldsymbol{u} \leq z / 2\}} \leq \sum_{i=1}^{d} A_{i},
$$

where

$$
A_{i}=\frac{\overline{H_{i}}\left(z_{i}-u_{i}\right)}{\overline{F_{i}}\left(t x_{i}\right)} 1_{\left\{0 \leq u_{i} \leq z_{i} / 2\right\}}
$$


3. Using (C4) for the marginals, as $t \rightarrow \infty$ we have

$$
A_{i} \rightarrow \beta_{i}\left(a_{i}+u_{i}\right)=\beta_{i}\left(a_{i}\right) \exp \left(\lambda(i) u_{i}\right) 1_{\left\{0 \leq u_{i}<\infty\right\}} .
$$

4. For the integral $T_{i}(1)=\int_{\mathbf{0}}^{z / 2} A_{i} \mathrm{~d} G(\boldsymbol{u})$, we have

$$
T_{i}(1)=\int_{0}^{z_{i} / 2} \frac{\overline{H_{i}}\left(z_{i}-u_{i}\right)}{\overline{F_{i}}\left(t x_{i}\right)} \mathrm{d} G_{i}\left(u_{i}\right)
$$

whence

$$
T_{i}(1)=\frac{\overline{F_{i}}\left(t x_{i}-a_{i}\right)}{\overline{F_{i}}\left(t x_{i}\right)} \int_{0}^{x_{i} / 2} \frac{\overline{H_{i}}\left(z_{i}-u_{i}\right)}{\overline{F_{i}}\left(z_{i}\right)} \mathrm{d} G_{i}\left(u_{i}\right) .
$$

Using $F_{i} \in S\left(\lambda_{i}\right)$ and Lemma 2 , we obtain

$$
T_{i}(1) \rightarrow \exp \left(\lambda(i) a_{i}\right) \beta_{i}(0) g_{i}(-\lambda(i))=\beta_{i}\left(a_{i}\right) g_{i}(-\lambda(i)),
$$

where $g_{i}(s)=\mathrm{E}\left(\exp \left(s U_{i}\right)\right)$. Now note that

$$
\beta_{i}\left(a_{i}\right) g_{i}(-\lambda(i))=\int_{0}^{\infty} \beta_{i}\left(a_{i}\right) \exp \left(\lambda(i) u_{i}\right) \mathrm{d} G_{i}\left(u_{i}\right)
$$

From Pratt's extension of Lebesgue's theorem on dominated convergence (see [31] or [20]), we conclude that

$$
T(1) \rightarrow \int_{\mathbf{0}}^{\infty} \beta(\boldsymbol{x}, \boldsymbol{a}+\boldsymbol{u}) \mathrm{d} G(\boldsymbol{u})=\mathrm{E}(\beta(\boldsymbol{x}, \boldsymbol{a}+\boldsymbol{U})) .
$$

We can treat the term corresponding to $J(2)$ in a similar way. The result follows.

(ii) The proof is similar to that of part (i).

\subsection{The asymptotic behaviour of $\bar{W}$ and $\overline{F^{* n}}$}

In the special case where $G=H$, Theorem 2 gives

$$
\lim _{t \rightarrow \infty} \frac{\overline{H * H}(t \boldsymbol{x}-\boldsymbol{a})}{\bar{F}(t \boldsymbol{x})}=2 \mathrm{E}(\beta(\boldsymbol{x}, \boldsymbol{a}+\boldsymbol{V}))=\beta_{2}(\boldsymbol{x}, \boldsymbol{a}) .
$$

Now note that $H * H$ is the DF of $\boldsymbol{V}_{1}+\boldsymbol{V}_{2}$, where $\boldsymbol{V}_{1}$ and $\boldsymbol{V}_{2}$ are independent copies of $\boldsymbol{V}$. Using Theorem 2 again, but now with $G=H * H$, we find that

$$
\lim _{t \rightarrow \infty} \frac{\overline{H^{* 3}}(t \boldsymbol{x}-\boldsymbol{a})}{\bar{F}(t \boldsymbol{x})}=\mathrm{E}\left(\beta_{2}(\boldsymbol{x}, \boldsymbol{V}+\boldsymbol{a})\right)+\mathrm{E}\left(\beta\left(\boldsymbol{x}, \boldsymbol{a}+\boldsymbol{V}_{1}+\boldsymbol{V}_{2}\right)\right)=\beta_{3}(\boldsymbol{x}, \boldsymbol{a}) .
$$

By continuing in this way, we obtain the following corollary.

Corollary 1. Suppose that (C2) and (C4) hold. Then, for all $n \geq 2$, we have

$$
\lim _{t \rightarrow \infty} \frac{\overline{H^{* n}}(t \boldsymbol{x}-\boldsymbol{a})}{\bar{F}(t \boldsymbol{x})}=\beta_{n}(\boldsymbol{x}, \boldsymbol{a}),
$$

where $\beta_{1}(\boldsymbol{x}, \boldsymbol{a})=\beta(\boldsymbol{x}, \boldsymbol{a}), \beta_{2}(\boldsymbol{x}, \boldsymbol{a})=2 \mathrm{E}\left(\beta\left(\boldsymbol{x}, \boldsymbol{a}+\boldsymbol{V}_{1}\right)\right)$, and

$$
\beta_{n+1}(\boldsymbol{x}, \boldsymbol{a})=\mathrm{E}\left(\beta_{n}(\boldsymbol{x}, \boldsymbol{V}+\boldsymbol{a})\right)+\mathrm{E}\left(\beta\left(\boldsymbol{x}, \boldsymbol{a}+\boldsymbol{V}_{1}+\boldsymbol{V}_{2}+\cdots+\boldsymbol{V}_{n}\right)\right) .
$$


Lemma 3 can be used to simplify this result. We replace $(\mathrm{C} 4)$ by the following condition.

(C5) Assume that $\bar{F} \in L^{*}\left(\mathbb{R}^{d}, v\right)$ and that, for all $\boldsymbol{a} \geq \mathbf{0}, \boldsymbol{b} \geq \mathbf{0}$, and $\boldsymbol{x}>\mathbf{0}$ with $x_{\min }<\infty$, we have

$$
\lim _{t \rightarrow \infty} \frac{\bar{H}(t \boldsymbol{x}-\boldsymbol{a}-\boldsymbol{b})}{\bar{F}(t \boldsymbol{x}-\boldsymbol{a})}=\beta(\boldsymbol{x}, \boldsymbol{b}) \text { for some real function } \beta .
$$

According to Lemma 3, we have $\beta(\boldsymbol{x}, \boldsymbol{b})=B(\boldsymbol{x}) v(\boldsymbol{x}, \boldsymbol{b})$.

For the limit functions $\beta_{n}$ appearing in Corollary 1 , we find that

$$
\begin{gathered}
\beta_{1}(\boldsymbol{x}, \boldsymbol{a})=B(\boldsymbol{x}) v(\boldsymbol{x}, \boldsymbol{a}), \quad \beta_{2}(\boldsymbol{x}, \boldsymbol{a})=2 B(\boldsymbol{x}) v(\boldsymbol{x}, \boldsymbol{a}) \mathrm{E}(v(\boldsymbol{x}, \boldsymbol{V})), \\
\mathrm{E}\left(\beta\left(\boldsymbol{x}, \boldsymbol{a}+\boldsymbol{V}_{1}+\boldsymbol{V}_{2}+\cdots+\boldsymbol{V}_{n}\right)\right)=B(\boldsymbol{x}) \mathrm{E}\left(\prod_{i=1}^{n} v\left(\boldsymbol{x}, \boldsymbol{V}_{i}\right)\right), \quad n \geq 2 .
\end{gathered}
$$

Because of independency,

$$
\mathrm{E}\left(\beta\left(\boldsymbol{x}, \boldsymbol{a}+\boldsymbol{V}_{1}+\boldsymbol{V}_{2}+\cdots+\boldsymbol{V}_{n}\right)\right)=B(\boldsymbol{x}) \mathrm{E}^{n}(v(\boldsymbol{x}, \boldsymbol{V})), \quad n \geq 2 .
$$

It follows that

$$
\beta_{n+1}(\boldsymbol{x}, \boldsymbol{a})=\mathrm{E}\left(\beta_{n}(\boldsymbol{x}, \boldsymbol{V}+\boldsymbol{a})\right)+B(\boldsymbol{x}) v(\boldsymbol{x}, \boldsymbol{a}) \mathrm{E}^{n}(v(\boldsymbol{x}, \boldsymbol{V})) .
$$

By induction on $n$ we find that

$$
\beta_{n}(\boldsymbol{x}, \boldsymbol{a})=n B(\boldsymbol{x}) v(\boldsymbol{x}, \boldsymbol{a}) \mathrm{E}^{n-1}(v(\boldsymbol{x}, \boldsymbol{V})), \quad n \geq 1 .
$$

We summarize our findings in the following result.

Corollary 2. Suppose that $\bar{F} \in L^{*}\left(\mathbb{R}^{d}, v\right)$ and that (C2) and (C5) hold. Then, for all $n \geq 1$, we have

$$
\lim _{t \rightarrow \infty} \frac{\overline{H^{* n}}(t \boldsymbol{x}-\boldsymbol{a})}{\bar{F}(t \boldsymbol{x})}=n B(\boldsymbol{x}) v(\boldsymbol{x}, \boldsymbol{a}) \mathrm{E}^{n-1}(v(\boldsymbol{x}, \boldsymbol{V})) .
$$

In the next result we generalize Theorem 1 . We use the notation $v_{n}(\boldsymbol{x}, \boldsymbol{a})$, similar to $\beta_{n}(\boldsymbol{x}, \boldsymbol{a})$ in Corollary 1. As in the introduction, $N$ denotes an integer-valued random variable with probability distribution $p_{n}=\mathrm{P}(N=n)$.

Theorem 3. (i) If (C1) and (C2) hold then, for all $\boldsymbol{x}>\mathbf{0}$ with $x_{\min }<\infty$ and all $\boldsymbol{a} \geq \mathbf{0}$, we have

$$
\lim _{t \rightarrow \infty} \frac{\overline{F^{* n}}(t \boldsymbol{x}-\boldsymbol{a})}{\bar{F}(t \boldsymbol{x})}=v_{n}(\boldsymbol{x}, \boldsymbol{a}), \quad n \geq 2 .
$$

(ii) If, for all $i=1,2, \ldots, d, P(z)=\mathrm{E}\left(z^{N}\right)$ is analytic at $f_{i}(-\lambda(i))$, then, for all $\boldsymbol{x}>\mathbf{0}$ with $x_{\min }<\infty$ and all $\boldsymbol{a} \geq \mathbf{0}$, we have

$$
\lim _{t \rightarrow \infty} \frac{\bar{W}(t \boldsymbol{x}-\boldsymbol{a})}{\bar{F}(t \boldsymbol{x})}=\sum_{n=1}^{\infty} p_{n} v_{n}(\boldsymbol{x}, \boldsymbol{a}) .
$$

(iii) If also $\bar{F} \in L^{*}\left(\mathbb{R}^{d}, v\right)$ then, for all $\boldsymbol{x}>\mathbf{0}$ with $x_{\min }<\infty$ and all $\boldsymbol{a} \geq \mathbf{0}$, we have

$$
\lim _{t \rightarrow \infty} \frac{\bar{W}(t \boldsymbol{x}-\boldsymbol{a})}{\bar{F}(t \boldsymbol{x})}=v(\boldsymbol{x}, \boldsymbol{a}) P^{\prime}(\mathrm{E}(v(\boldsymbol{x}, \boldsymbol{x}))) .
$$


Proof. Only (ii) needs an explicit proof. First observe that

$$
\bar{W}(t \boldsymbol{x}-\boldsymbol{a})=\sum_{n=1}^{\infty} p_{n} \overline{F^{* n}}(t \boldsymbol{x}-\boldsymbol{a}) .
$$

To apply Lebesgue's theorem on dominated convergence, note that

$$
\overline{F^{* n}}(t \boldsymbol{x}-\boldsymbol{a}) \leq \sum_{i=1}^{d} \overline{F_{i}^{* n}}\left(t x_{i}-a_{i}\right) .
$$

It is well known that, since $F_{i} \in S_{\lambda(i)}$ for each $\varepsilon>0$, we can find constants $u_{i}, v_{i}>0$ such that

$$
\overline{F_{i}^{* n}}\left(t x_{i}-a_{i}\right) \leq u_{i}\left(f_{i}(-\lambda(i))+\varepsilon\right)^{n} \overline{F_{i}}\left(t x_{i}\right), \quad t>v_{i} .
$$

Using $\overline{F_{i}}\left(t x_{i}\right) \leq \bar{F}(t \boldsymbol{x})$, we have $\overline{F_{i}^{* n}}\left(t x_{i}-a_{i}\right) \leq u_{i}\left(f_{i}(-\lambda(i))+\varepsilon\right)^{n} \bar{F}(t \boldsymbol{x})$ for all $t>v_{i}$. It follows that we can find constants $u, v>0$ such that

$$
\overline{F^{* n}}(t \boldsymbol{x}-\boldsymbol{a}) \leq u \sum_{i=1}^{d}\left(f_{i}(-\lambda(i))+\varepsilon\right)^{n} \bar{F}(t \boldsymbol{x}), \quad t>v .
$$

Part (ii) now follows from Lebesgue's theorem on dominated convergence.

\subsection{Two-dimensional subordination}

In the previous sections we investigated $\overline{F^{* n}}$ and $\bar{W}$ under a variety of conditions. In this section we consider random vectors of the form

$$
\boldsymbol{S}(\boldsymbol{N})=\left(S^{1}\left(N_{1}\right), S^{2}\left(N_{2}\right), \ldots, S^{d}\left(N_{d}\right)\right)
$$

and study the asymptotic behaviour of $\bar{K}$, where $K(\boldsymbol{x})=\mathrm{P}(\boldsymbol{S}(\boldsymbol{N}) \leq \boldsymbol{x})$.

For simplicity, we restrict our attention to the two-dimensional case. To start our analysis, consider independent, identically distributed copies, $Z_{1}=\left(X_{1}, Y_{1}\right), Z_{2}=\left(X_{2}, Y_{2}\right), \ldots$, $Z_{n}=\left(X_{n}, Y_{n}\right)$, of $Z=(X, Y)$. Taking partial sums, we define

$$
F_{m, n}(x, y)=\mathrm{P}\left(\sum_{i=1}^{m} X_{i} \leq x, \sum_{i=1}^{n} Y_{i} \leq y\right) .
$$

For $n=0$ and $m \geq 1$, we define $F_{0, m}(x, y)=\mathrm{P}\left(\sum_{i=1}^{m} Y_{i} \leq y\right)=F_{2}^{* m}(y)$ and $F_{m, 0}(x, y)=$ $\mathrm{P}\left(\sum_{i=1}^{m} X_{i} \leq x\right)=F_{1}^{* m}(x)$. Finally, we set $F_{0,0}(x, y)=1$. Note that $F^{* n}(x, y)=F_{n, n}(x, y)$. Theorem 4 shows that if (C1) and (C2) hold, then

$$
\frac{\overline{F_{n, n}}(t x-a, t y-b)}{\bar{F}(t x, t y)} \rightarrow v_{n}((x, y),(a, b)) .
$$

In the next result we consider $F_{m, n}(x, y)$. To prove the result we need the following additional assumption.

(C6) For $i=1,2$, there exist real functions $D_{i}(x, y)$ such that, for all $(x, y)>(0,0)$ with $\min (x, y)<\infty$, we have

$$
\frac{\overline{F_{i}}(t x)}{\bar{F}(t x, t y)} \rightarrow D_{i}(x, y) .
$$


Proposition 1. Suppose that $F \in L^{*}\left(\mathbb{R}^{2}, v\right)$ and that (C2) and (C6) hold. Then

$$
\lim _{t \rightarrow \infty} \frac{1-F_{m, n}(t x-a, t y-b)}{\bar{F}(t x, t y)}=L(m, n), \quad m, n \geq 1,
$$

where $L(m, n)$ will be determined in the proof of the result.

Proof. Suppose that $m>n$ and set

$$
\boldsymbol{V}=\left(\sum_{i=1}^{n} X_{i}, \sum_{i=1}^{n} Y_{i}\right) \text { and }(U, 0)=\left(\sum_{i=n+1}^{m} X_{i}, 0\right)
$$

with DFs $H=F^{* m}(x, y)$ and $G(x, y)$, respectively. Clearly $\bar{F}_{m, n}(t x-a, t y-b)=$ $\overline{G * H}(t x-a, t y-b)$.

For the vector $\boldsymbol{V}$, conditions $(\mathrm{C} 1)$ and (C2) show that

$$
\frac{\bar{H}(t x-a, t y-b)}{\bar{F}(t x, t y)} \rightarrow v_{n}((x, y),(a, b)) .
$$

To treat $G(\cdot, \cdot)$, note that

$$
G(x, y)=\mathrm{P}\left(\sum_{i=n+1}^{m} X_{i} \leq x, 0 \leq y\right)=F_{1}^{*(m-n)}(x)
$$

and that $\bar{G}(x, y)=\overline{F_{1}^{*(m-n)}}(x)$. Using (C2), the univariate results show that

$$
\lim _{x \rightarrow \infty} \frac{\bar{G}(x, y)}{\overline{F_{1}}(x)} \rightarrow(m-n) \delta_{1}^{m-n-1},
$$

where $\delta_{1}=f_{1}(-\lambda(1))$. It follows that

$$
\lim _{t \rightarrow \infty} \frac{\bar{G}(t x-a, t y-b)}{\overline{F_{1}}(t x)} \rightarrow(m-n) \delta_{1}^{m-n-1} c_{1}(a),
$$

where $c_{1}(a)=\exp (\lambda(1) a)$. Using (C6), we find that

$$
\lim _{x \rightarrow \infty} \frac{\bar{G}(t x-a, t y-b)}{\bar{F}(t x, t y)} \rightarrow(m-n) \delta_{1}^{m-n-1} c_{1}(a) D_{1}(x, y) .
$$

Theorem 2 now yields

$$
\frac{1-F_{m, n}(t x-a, t y-b)}{\bar{F}(t x, t y)} \rightarrow L_{1}(m, n), \quad m>n,
$$

where

$$
\begin{aligned}
L_{1}(m, n)= & (m-n) \delta_{1}^{m-n-1} D_{1}(x, y) \mathrm{E}\left(c_{1}\left(a+\sum_{i=1}^{n} X_{i}\right)\right) \\
& +\mathrm{E}\left(v_{n}\left((x, y),\left(a+\sum_{i=n+1}^{m} X_{i}, b\right)\right)\right) .
\end{aligned}
$$


In a similar way, for $n>m$ we find that

$$
\frac{1-F_{m, n}(t x-a, t y-b)}{\bar{F}(t x, t y)} \rightarrow L_{2}(m, n), \quad n>m,
$$

where

$$
\begin{aligned}
L_{2}(m, n)= & (n-m) \delta_{2}^{n-m-1} D_{2}(x, y) \mathrm{E}\left(c_{2}\left(b+\sum_{i=1}^{m} Y_{i}\right)\right) \\
& +\mathrm{E}\left(v_{m}\left((x, y),\left(a, b+\sum_{i=m+1}^{n} Y_{i}\right)\right)\right) .
\end{aligned}
$$

In order to simplify the complicated expressions for $L_{1}(m, n)$ and $L_{2}(m, n)$, first observe that $c_{1}(a)=\exp (\lambda(1) a)$. It follows that

$$
\mathrm{E}\left(c_{1}\left(a+\sum_{i=1}^{n} X_{i}\right)\right)=c_{1}(a) \mathrm{E}^{n}(\exp (\lambda(1) X))=c_{1}(a) \delta_{1}^{n} .
$$

In a similar way we find that $\mathrm{E}\left(c_{2}\left(b+\sum_{i=1}^{m} Y_{i}\right)\right)=c_{2}(b) \delta_{2}^{m}$. To simplify the second term in the formula for $L_{1}$ we use the fact that $F \in L^{*}\left(\mathbb{R}^{2}, v\right)$ and the relation $v(\boldsymbol{x}, \boldsymbol{a}+\boldsymbol{b})=v(\boldsymbol{x}, \boldsymbol{a}) v(\boldsymbol{x}, \boldsymbol{b})$. Earlier (see Corollary 2) we found that

$$
v_{n}((x, y),(a, b))=n v((x, y),(a, b)) \mathrm{E}^{n-1}(v((x, y),(X, Y))), \quad n \geq 1 .
$$

Using this relation, we find that

$$
\begin{aligned}
& \mathrm{E}\left(v_{n}\left((x, y),\left(a+\sum_{i=n+1}^{m} X_{i}, b\right)\right)\right) \\
& \quad=n v((x, y),(a, b)) \mathrm{E}^{n-1}(v((x, y),(X, Y))) \mathrm{E}^{m-n}(v((x, y),(X, 0))) .
\end{aligned}
$$

In a similar way we find that

$$
\begin{aligned}
& \mathrm{E}\left(v_{m}\left((x, y),\left(a, b+\sum_{i=m+1}^{n} Y_{i}\right)\right)\right) \\
& \quad=m v((x, y),(a, b)) \mathrm{E}^{m-1}(v((x, y),(X, Y))) \mathrm{E}^{n-m}(v((x, y),(0, Y))) .
\end{aligned}
$$

If $F \in L\left(\mathbb{R}^{2}\right)$ we can simplify even more, to obtain

$$
L_{1}(m, n)=(m-n) D_{1}(x, y)+n \quad \text { and } \quad L_{2}(m, n)=(n-m) D_{2}(x, y)+m .
$$

Corollary 3. If $F \in L\left(\mathbb{R}^{2}\right)$ and satisfies (C2) and (C6), then

$$
\lim _{t \rightarrow \infty} \frac{1-F_{m, n}(t x-a, t y-b)}{\bar{F}(t x, t y)}=L(m, n), \quad m, n \geq 1,
$$

where

$$
L(m, n)= \begin{cases}(m-n) D_{1}(x, y)+n, & m \geq n \\ (n-m) D_{2}(x, y)+m, & n \geq m .\end{cases}
$$


We now use this result in the context of random sums of random vectors. Let $\left(N_{1}, N_{2}\right)$ denote a pair of integer-valued random variables independent of $(X, Y)$. The DF of the random vector $\left(S^{1}\left(N_{1}\right), S^{2}\left(N_{2}\right)\right)$ is given by

$$
G(x, y)=\sum_{m=0}^{\infty} \sum_{n=0}^{\infty} \mathrm{P}\left(N_{1}=m, N_{2}=n\right) F_{m, n}(x, y) .
$$

We have

$$
\bar{K}(x, y)=\sum_{m=0}^{\infty} \sum_{n=0}^{\infty} \mathrm{P}\left(N_{1}=m, N_{2}=n\right)\left(1-F_{m, n}(x, y)\right) .
$$

Following the proof of Theorem 3(ii), we obtain the following result.

Theorem 4. Suppose that $F \in L^{*}\left(\mathbb{R}^{2}, v\right)$ and satisfies (C2) and (C6). Suppose that $\mathrm{E}\left(z^{N_{i}}\right)$ is analytic at $z=f(-\lambda(i))$ for $i=1,2$. Then

$$
\lim _{t \rightarrow \infty} \frac{\bar{K}(t x-a, t y-b)}{\bar{F}(t x, t y)}=\mathrm{E}\left(L\left(N_{1}, N_{2}\right)\right) .
$$

As a special case we have the following result.

Corollary 4. Suppose that $F \in L\left(\mathbb{R}^{2}\right)$ and satisfies $(C 2)$ and (C6). Suppose that $\mathrm{E}\left(z^{N_{i}}\right)$ is analytic at $z=1$ for $i=1,2$. Then

$$
\lim _{t \rightarrow \infty} \frac{\bar{K}(t x-a, t y-b)}{\bar{F}(t x, t y)}=\mathrm{E}\left(L\left(N_{1}, N_{2}\right)\right)
$$

with

$$
\mathrm{E}\left(L\left(N_{1}, N_{2}\right)\right)=D_{1}(x, y) \mathrm{E}\left(\left(N_{1}-N_{2}\right)^{+}\right)+D_{2}(x, y) \mathrm{E}\left(\left(N_{2}-N_{1}\right)^{+}\right)+\mathrm{E}\left(\min \left(N_{1}, N_{2}\right)\right),
$$

where $N^{+}=\max (0, N)$.

\section{Relation with multivariate regular variation}

In the univariate case, we can prove that regular variation of $\bar{F}$ implies that $F \in S$ and that $\bar{F} \in L$. In the multivariate case, a similar result holds. The following classes of functions have been introduced and studied by Stam [36], Resnick [32], Omey [25], [26], and de Haan et al. [10], [11].

In what follows, for $i=1,2, \ldots, d$ we assume that $c_{i}(t) \in R V\left(\alpha_{i}\right), \alpha_{i}>0$, and we let $h(t)$ denote a positive, measurable function. Let $u: \mathbb{R}^{d} \rightarrow \mathbb{R}$ and, as before, let $c * \boldsymbol{x}=$ $\left(c_{1}(t) x_{1}, c_{2}(t) x_{2}, \ldots, c_{d}(t) x_{d}\right)$. Recall that $f(x) \in R V(\alpha)$, the class of functions that are regularly varying with index $\alpha$, if and only if $\lim _{t \rightarrow \infty} f(t x) / f(t)=x^{\alpha}$ for all $x>0$.

Definition 7. Let $\boldsymbol{x}$ denote a positive, $d$-dimensional random vector with DF $F(\boldsymbol{x})$ and let $h(t) \in R V(-\beta)$ for some $\beta \geq 0$. If $\beta=0$, also assume that $h(t) \rightarrow 0$. In this case, we say that $\bar{F}(\boldsymbol{x}) \in R V F(\boldsymbol{c}, h)$ if, for all $\boldsymbol{x}>\mathbf{0}$ with $x_{\min }<\infty$, we have

$$
\lim _{t \rightarrow \infty} \frac{\bar{F}(\boldsymbol{c}(t) * \boldsymbol{x})}{h(t)}=\mu(\boldsymbol{x})<\infty .
$$


Since $\bar{F}(\boldsymbol{x})$ is monotone, the limit function $\mu(\boldsymbol{x})$ is continuous and the convergence is locally uniform in $\boldsymbol{x}$ (see [26, p. 17]). It follows that

$$
\lim _{t \rightarrow \infty} \frac{\bar{F}(\boldsymbol{c}(t) * \boldsymbol{x}-\boldsymbol{a})}{h(t)}=\mu(\boldsymbol{x})<\infty
$$

for all $\boldsymbol{x}>\mathbf{0}$ with $x_{\min }<\infty$ and all $\boldsymbol{a} \geq \mathbf{0}$. If $\mu(\boldsymbol{x})>0$ then $\bar{F}(\boldsymbol{x}) \in L^{c}\left(\mathbb{R}^{d}, v=1\right)$. If $c_{i}(t)=t, i=1,2, \ldots, d$, then the following result, proved by Omey [27], holds. If $\mu(\boldsymbol{x})>0$ then the result follows from Theorem 5 .

Theorem 5. Suppose that $h(t) \in R V(-\beta)$ for some $\beta>0$ and suppose that $\bar{F}(\boldsymbol{x}) \in$ $R V F(\boldsymbol{c}, h)$ with limit function $\mu(\boldsymbol{x})$.

(i) for all $n \geq 1$ and all $\boldsymbol{x}>\mathbf{0}$ with $x_{\min }<\infty$, we have

$$
\lim _{t \rightarrow \infty} \frac{\overline{F^{* n}}(\boldsymbol{c}(t) * \boldsymbol{x})}{h(t)}=n \mu(\boldsymbol{x}) .
$$

(ii) If $P(z)$ is analytic at $z=1$ then, for all $\boldsymbol{x}>\mathbf{0}$ with $x_{\min }<\infty$, we have

$$
\lim _{t \rightarrow \infty} \frac{\bar{W}(\boldsymbol{c}(t) * \boldsymbol{x})}{h(t)}=\mathrm{E}(N) \mu(\boldsymbol{x}) .
$$

Remarks 3. (i) As an alternative, we can work with the 'tail' $\mathrm{P}(\boldsymbol{X}>\boldsymbol{x})$ and define regular variation by assuming that

$$
\lim _{t \rightarrow \infty} \frac{\mathrm{P}(\boldsymbol{X}>t \boldsymbol{x})}{h(t)}=\lambda(\boldsymbol{x})<\infty \quad \text { for all } \boldsymbol{x} \geq \mathbf{0} \text { with } \max \left(x_{i}\right)>0 .
$$

Again convergence is locally uniform and if $\lambda(\boldsymbol{x})>0$ then $\mathrm{P}(\boldsymbol{X}>\boldsymbol{x}) \in L\left(\mathbb{R}^{d}, v=1\right)$. In this case, Omey [27] showed that $\mathrm{P}(\boldsymbol{S}(n)>t \boldsymbol{x}) / h(t) \rightarrow n \lambda(\boldsymbol{x})$.

(ii) Recently, Basrak et al. [2] proved that if $\bar{F}(x) \in R V F(\boldsymbol{c}, h)$, where $h(t) \in R V(-\beta)$ with $\beta>0, c_{i}(t)=t$ for $1 \leq i \leq d$, and $\mu(\boldsymbol{x})>0$, then

$$
\begin{aligned}
\lim _{u \rightarrow \infty} \frac{\mathrm{P}((\boldsymbol{x}, \boldsymbol{X})>u)}{h(u)}= & w(\boldsymbol{x})<\infty \quad \text { for all } \boldsymbol{x} \in \mathbb{R}^{d}, \\
& w(\boldsymbol{y})>0 \quad \text { for at least one } \boldsymbol{y} \in \mathbb{R}^{d} .
\end{aligned}
$$

Here $(\boldsymbol{a}, \boldsymbol{b})=\sum_{i=1}^{d} a_{i} b_{i}$. Conversely, if

$$
\left.\lim _{u \rightarrow \infty} \frac{\mathrm{P}((\boldsymbol{x}, \boldsymbol{X})>u)}{h(u)}=w(\boldsymbol{x})<\infty \quad \text { for all } \boldsymbol{x} \geq \mathbf{0} \text { (but } \boldsymbol{x} \neq \mathbf{0}\right)
$$

with $\beta>0$ a noninteger, then $\bar{F}(\boldsymbol{x}) \in R V F(\boldsymbol{c}, h)$ with $c_{i}(t)=t, 1 \leq i \leq d$. In the case where $\beta$ is an integer, the converse is in general false [19].

(iii) The class $R V F$ has proved to be useful in limit theory in probability theory and in number theory, having applications in, for example, extreme value theory, domains of attraction for sums of independent, identically distributed random vectors, renewal theory, and generalized renewal theory. For a survey of properties and applications in probability theory, we refer 
the reader to [10], [11], [32], and [33]. In [30] several Abelian and Tauberian theorems were obtained, and the results applied in summability theory (see also [1]). Basrak et al. [2] used $R V F$ functions to obtain new results for linear stochastic recurrence relations and for generalized autoregressive conditionally heteroscedastic processes. Here we use such functions to obtain some new results in multivariate shock models (see [21] and [23]).

\section{Applications and concluding remarks}

\subsection{Applications}

Random vectors of the form $\left(S^{1}\left(N_{1}\right), S^{2}\left(N_{2}\right)\right)$ can be interpreted in insurance portfolios as follows. Suppose that we have a sequence of independent, identically distributed random vectors $\left(X_{i}, Y_{i}\right), i=1,2, \ldots$, where $X_{i}$ and $Y_{i}$ denote the claim sizes of claims of type 1 and type 2 , respectively. We can think of accidents which involve personal and/or material damage. At time $t>0$, the number of claims of type $i$ is given by $N_{i}(t)$ and the total claim size is given by $\left(S^{1}\left(N_{1}\right), S^{2}\left(N_{2}\right)\right)$. Our results can be used to estimate $\mathrm{P}\left(\max \left(S^{1}\left(N_{1}\right), S^{2}\left(N_{2}\right)\right)>x\right)$ for large values of $x$.

Another interpretation comes from reliability theory. Suppose that a material system is subject to shocks that have multidimensional effects. One shock may, for example, influence both the temperature and the strength of the material. Suppose that the $i$ th shock results in a vector of damage given by $\left(X_{i}, Y_{i}\right)$. If the number of shocks is given by $N$, then the accumulated damage is given by the vector $\left(S^{1}(N), S^{2}(N)\right)$. Our results can be used to estimate $\mathrm{P}\left(S^{1}(N) \leq t x, S^{2}(N) \leq t y\right)$ for large values of $t$. In [24] the present authors studied renewal quantities related to this type of process.

We can also use our results to study the multivariate analogues of [21], [22] and [34]. In this case, we study a sequence of random vectors $\boldsymbol{Y}_{k}$ defined as follows. Let $\boldsymbol{A}, \boldsymbol{B}$, and $\boldsymbol{C}$ denote random variables with generating functions given by $a(s), b(s)$, and $c(s)$, respectively. Let $p$ and $q$ denote real numbers such that $0<p=1-q<1$ and $b(\boldsymbol{s})=p a(s)+q c(s)$. The sequence $\boldsymbol{Y}_{k}$ is defined recursively using the following generating functions:

$$
g_{0}(\boldsymbol{s})=1, \quad g_{k}(\boldsymbol{s})=\frac{p a(s) g_{k-1}(\boldsymbol{s})}{1-q c(\boldsymbol{s}) g_{k-1}(\boldsymbol{s})}, \quad k=1,2, \ldots
$$

Using a Taylor expansion, we find that

$$
g_{k}(\boldsymbol{s})=a(\boldsymbol{s}) g_{k-1}(\boldsymbol{s}) \sum_{n=0}^{\infty} p q^{n}\left(c(\boldsymbol{s}) g_{k-1}(\boldsymbol{s})\right)^{n} .
$$

Clearly $\boldsymbol{Y}_{k}$ is related to a compound geometric sum as follows. Let $N$ denote a geometric random variable with $\mathrm{P}(N=n)=p q^{n}, n \geq 0$. For $i \geq 1$, let $\boldsymbol{Y}_{k}(i)$ denote independent, identically distributed copies of $\boldsymbol{Y}_{k}$ and, independently, let $\boldsymbol{C}_{i}$ denote independent, identically distributed copies of $\boldsymbol{C}$. Define $\boldsymbol{S}_{k-1}(n)$ by

$$
S_{k-1}(0)=0, \quad S_{k-1}(n)=\sum_{i=1}^{n} \boldsymbol{Y}_{k-1}(i) .
$$

From (3) we see that $\boldsymbol{Y}_{k} \stackrel{\mathrm{D}}{=} \boldsymbol{A}+\boldsymbol{Y}_{k-1}+\boldsymbol{S}_{k-1}(N)$. Our results can be used to study the asymptotic behaviour of $1-\mathrm{P}\left(\boldsymbol{Y}_{k} \leq \boldsymbol{x}\right)$. As in the univariate case, the random variable $\boldsymbol{Y}_{k}$ can be interpreted in terms of shock models or insurance mathematics. 


\subsection{Concluding remarks}

(i) For $\mu(x)>0$ and $c=t$, Theorem 1 shows that

$$
\overline{F^{* n}}(t \boldsymbol{x}) \sim n \bar{F}(t \boldsymbol{x}) .
$$

Relation (4) admits the following probabilistic interpretation. Since $n(1-F(t \boldsymbol{x})) \sim 1-F^{n}(t \boldsymbol{x})$ and $\overline{F^{* n}}(t \boldsymbol{x}) \sim n \bar{F}(t \boldsymbol{x}),(4)$ implies that

$$
1-\mathrm{P}(\boldsymbol{S}(n) \leq t \boldsymbol{x}) \sim 1-\mathrm{P}(\boldsymbol{M}(n) \leq t \boldsymbol{x}),
$$

where $S(n)=\left(S^{1}(n), S^{2}(n), \ldots, S^{d}(n)\right)$ denotes the vector of partial sums and $\boldsymbol{M}(n)=$ $\left(M^{1}(n), M^{2}(n), \ldots, M^{d}(n)\right)$ denotes the vector of partial maxima. Clearly

$$
\begin{gathered}
1-\mathrm{P}(\boldsymbol{S}(n) \leq t \boldsymbol{x})=\mathrm{P}\left(\max _{i} \frac{S^{i}(n)}{x_{i}}>t\right), \\
1-\mathrm{P}(\boldsymbol{M}(n) \leq t \boldsymbol{x})=\mathrm{P}\left(\max _{i} \frac{M^{i}(n)}{x_{i}}>t\right) .
\end{gathered}
$$

It follows that

$$
\mathrm{P}\left(\max _{i} \frac{S^{i}(n)}{x_{i}}>t\right) \sim \mathrm{P}\left(\max _{i} \frac{M^{i}(n)}{x_{i}}>t\right) .
$$

In the univariate case, we know that the tails of $S^{i}(n)$ and $M^{i}(n)$ are asymptotically the same. In the multivariate case, the previous result shows that $\max _{i}\left(S^{i}(n) / x_{i}\right)$ and $\max _{i}\left(M^{i}(n) / x_{i}\right)$ also have the same tail behaviour. It would be interesting to study the relation between the tail behaviours of

$$
\min _{i} \frac{S^{i}(n)}{x_{i}}, \quad \max _{1 \leq j \leq n} \min _{1 \leq i \leq d} \frac{X_{j}^{i}}{x_{i}}, \quad \sum_{j=1}^{n} \min _{i} \frac{X_{j}^{i}}{x_{i}}, \quad \text { and } \quad \min _{1 \leq i \leq d} \max _{1 \leq j \leq n} \frac{X_{j}^{i}}{x_{i}} .
$$

It would also be interesting to study these quantities for triangular arrays.

(ii) The condition $\bar{F} \in L\left(\mathbb{R}^{d}\right)$ can be reformulated as follows. Let $\boldsymbol{X}=\left(X_{1}, X_{2}, \ldots, X_{d}\right)$ denote a positive random variable with DF $F(\boldsymbol{x})$. For a fixed $\boldsymbol{x}=\left(x_{1}, x_{2}, \ldots, x_{d}\right)>\mathbf{0}$, define the new random variable $Z(\boldsymbol{x})=\max \left(X_{i} / x_{i}, i=1,2, \ldots, d\right)$. Clearly the DF of $Z(\boldsymbol{x})$ is given by $\mathrm{P}(Z(x) \leq t)=F_{Z(x)}(t)=F(t \boldsymbol{x})$. Since $\bar{F}$ is monotone, it is easy to show that $\bar{F}(\boldsymbol{x}) \in L\left(\mathbb{R}^{d}\right)$ if and only $\bar{F}_{Z(\boldsymbol{x})}(t) \in L(\mathbb{R})$. Note that Theorem 1 shows that $\bar{F}_{Z(\boldsymbol{x})}(t) \in L(\mathbb{R})$ implies $F \in S\left(\mathbb{R}^{d}\right)$.

(iii) In this paper, among others, the present authors have studied the asymptotic behaviour of sums of the form $W(\boldsymbol{x})=\sum_{n=0}^{\infty} p_{n} F^{* n}(\boldsymbol{x})$, where $\left\{p_{n}\right\}$ denotes a probability distribution. It is also possible to study $W(\boldsymbol{x})$ using sequences $\left\{p_{n}\right\}$ of other sorts. For example, if $p_{n}=1$ then we obtain the $d$-dimensional renewal function (see [30]). If $p_{0}=0$ and $p_{n}=1 / n, n \geq 1$, then we obtain the harmonic renewal function. In [29] and [24], generalized renewal functions in $\mathbb{R}$ and $\mathbb{R}^{d}$ were studied.

(iv) The concept of subexponential densities in $\mathbb{R}^{d}$ has not been studied yet. For densities, subexponentiality could be defined by assuming that $f(\boldsymbol{x}) \in L\left(\mathbb{R}^{d}\right)$ and that

$$
\frac{f \otimes f(t \boldsymbol{x})}{f(t \boldsymbol{x})} \rightarrow \alpha(\boldsymbol{x})
$$

for some limit function. More research is needed in this area. 


\section{Acknowledgement}

Part of this paper was written while E. Omey visited the Public University of Navarre. He takes pleasure in thanking the university for its hospitality.

\section{References}

[1] Alpár, L. (1984). Tauberian theorems for power series of two variables. Studia Sci. Math. Hung. 19, 165-176.

[2] Basrak, B., Davis, R. A. AND Mikosch, T. (2002). A characterization of multivariate regular variation. Ann. Appl. Prob. 12, 908-920.

[3] Bingham, N. H., Goldie, C. M. and Teugels, J. L. (1987). Regular Variation (Encyclopaedia Math. Appl. 27). Cambridge University Press.

[4] Chistyakov, V. P. (1964). A theorem on sums of independent positive random variables and its applications to branching random processes. Theory Prob. Appl. 9, 640-648.

[5] Chover, J., Ney, P. And Wainger, S. (1973). Degeneracy properties of subcritical branching processes. Ann. Prob. 1, 663-673.

[6] Chover, J., Ney, P. and Wainger, S. (1973). Functions of probability measures. J. Anal. Math. 26, 255-302.

[7] Cline, D. B. H. (1986). Convolution tails, product tails and domains of attraction. Prob. Theory Relat. Fields 72, 529-557.

[8] Cline, D. B. H. (1987). Convolutions of distributions with exponential and subexponential tails. J. Austral. Math. Soc. A 43, 347-365. (Correction: 48 (1990), 152-153.)

[9] Cline, D. B. H. and Resnick, S. I. (1992). Multivariate subexponential distributions. Stoch. Process. Appl. 42, 49-72.

[10] De HaAn, L. ANd OMEy, E. (1984). Integrals and derivatives of regularly varying functions in $\mathbb{R}^{d}$ and domains of attraction of stable distributions. II. Stoch. Process. Appl. 16, 157-170.

[11] De HaAn, L., Omey, E. And Resnick, S. I. (1984). Domains of attraction and regular variation in $\mathbb{R}^{d}$. J. Multivariate Anal. 14, 17-33.

[12] Embrechts, P. (1985). Subexponential distribution functions and their applications: a review. In Proc. 7th Conf. Prob. Theory (Brasov, Romania, 1982), eds M. Iosifescu et al., VSP, Zeist, pp. 125-136.

[13] Embrechts, P. AND Goldie, C. M. (1980). On closure and factorization properties of subexponential and related distributions. J. Austral. Math. Soc. A 29, 243-256.

[14] Embrechts, P. And Goldie, C. M. (1982). On convolution tails. Stoch. Process. Appl. 13, 263-278.

[15] Embrechts, P., Goldie, C. M. and Veraverbeke, N. (1979). Subexponentiality and infinite divisibility. $Z$. Wahrscheinlichkeitsth. 49, 335-347.

[16] Embrechts, P., Klüppelberg, C. And Mikosch, T. (1997). Modelling Extremal Events (Appl. Math. 33). Springer, New York.

[17] Geluk, J. L. And De HaAn, L. (1987). Regular Variation, Extensions and Tauberian Theorems (CWI Tracts 40). Centre For Mathematics and Computer Science, Amsterdam.

[18] Goldie, C. M. And Klüppelberg, C. (1998). Subexponential distributions. In A Practical Guide to Heavy Tails, eds R. J. Adler, R. E. Feldman and M. S. Taqqu, Birkhäuser, Boston, MA, pp. 435-459.

[19] Hult, H. And Linskog, F. (2006). On Kesten's counterexample to the Cramér-Wold device for regular variation. Bernoulli 12, 133-142.

[20] Johns, M. V., JR. (1957). Non-parametric empirical Bayes procedures. Ann. Math. Statist. 28, 649-669.

[21] Mallor, F. And Omey, E. (2001). Shocks, runs and random sums. J. Appl. Prob. 38, 438-448.

[22] Mallor, F. AND OMey, E. (2002). Shocks, runs and random sums: asymptotic behaviour of the distribution function. J. Math. Sci. NY 111, 3559-3565.

[23] Mallor, F., Omey, E. And Santos, J. (2006). Asymptotic results for a run and cumulative mixed shock model. J. Math. Sci. 138, 5410-5415.

[24] Mallor, F., Omey, E. And Santos, J. (2007). Multivariate weighted renewal functions. J. Multivariate Anal. 98, 30-39.

[25] Omey, E. (1982). Multivariate Reguliere Variatie en Toepassingen in Kanstheorie. Doctoral Thesis. Katholieke Universiteit Leuven.

[26] OMey, E. (1989). Multivariate Regular Variation and Applications in Probability Theory (Eclectica 74). EHSAL, Brussels.

[27] Омеy, E. (1990). Random sums of random vectors. Publ. Inst. Math. 48, 191-198.

[28] OMey, E. (2006). Subexponential distributions and the difference between the product and the convolution product of distribution functions in $\mathbb{R}^{d}$. J. Math. Sci. 138, 5434-5449.

[29] Omey, E. And Teugels, J. L. (2002). Weighted renewal functions: a hierarchical approach. Adv. Appl. Prob. 34, 394-415. 
[30] Omey, E. AND Willekens, E. (1989). Abelian and Tauberian theorems for the Laplace transform of functions in several variables. J. Multivariate Anal. 30, 292-306.

[31] Pratt, J. W. (1960). On interchanging limits and integrals. Ann. Math. Statist. 31, 74-77.

[32] Resnick, S. I. (1986). Point processes, regular variation and weak convergence. Adv. Appl. Prob. 18, 66-138.

[33] Resnick, S. I. (1987). Extreme Values, Regular Variation and Point Processes (Appl. Prob. 4). Springer, New York.

[34] Santos, J. (2004). Fiabilidad de sistemas sometidos a choques. Doctoral Thesis, Public University of Navarre, Pamplona.

[35] Seneta, E. (1976). Regularly Varying Functions (Lecture Notes Math. 508). Springer, New York.

[36] Stam, A. J. (1977). Regular variation in $R_{+}^{d}$ and the Abel-Tauber theorem. Tech. Rep. TW 189, Mathematics Institute, University of Groningen.

[37] Teugels, J. L. (1975). The class of subexponential distributions. Ann. Prob. 3, 1000-1011. 